\title{
Improving road-side surveys for a better knowledge of road freight transport
}

\author{
François Combes • Fabien Leurent
}

Received: 19 October 2010 / Accepted: 10 October 2011 / Published online: 11 September 2012

(C) The Author(s) 2012. This article is published with open access at SpringerLink.com

\begin{abstract}
Purpose The focus of this paper is to enhance our observation and knowledge of interurban road freight transport. It explores some possibilities for improving roadside freight surveys, usually used to gather origindestination data.

Methodology To achieve this, new questions are added to a standard roadside survey form, and the enhanced survey form is tested through two surveys. The new questions relate to currently unobserved variables: the vehicle volume occupied by freight; the method of organisation (double crew, relays) used by the carriers; the existence of specific logistical imperatives; and drivers' compulsory breaks.

Results The questions on volume constraint and carrier organisation prove to be the most informative. These two questions are thus two promising areas for improvement in roadside freight surveys. The questions about specific logistical imperatives and driver breaks prove less fruitful.

Conclusion The paper concludes that it is possible, through minor modifications in data collection protocols, to significantly enhance observation quality in road freight transport activity.
\end{abstract}

Keywords Freight transport • Truck size and weight • Roadside survey $\cdot$ Transport organisation

F. Combes $(\bowtie) \cdot$ F. Leurent

Université Paris-Est, LVMT, UMR T9403 INRETS ENPC

UMLV, 6-8 av. Blaise Pascal, Cité Descartes, 77455

Champs-sur-Marne, Marne-la-Vallée Cedex 2, France

e-mail: francois.combes@enpc.fr

F. Leurent

e-mail: fabien.leurent@enpc.fr

\section{Introduction}

Theoretical models, simulation models and various kinds of quantitative and qualitative analyses of road freight transport rely on databases describing the activity of road freight transport systems. These databases consist of observations obtained through data collection protocols. Many types of data collection protocols exist; they can be grouped into the following categories [1]: roadside intercepts, telephone interviews, mailout/mail-back, combined telephone and mail-back, personal interviews, internet, focus and stakeholder groups, commercial vehicle trip diaries, Global Positioning System (GPS) vehicle tracking, license plate match, and administrative surveys. Of course, each of these protocols has its strengths and weaknesses, and is therefore useful in different ways.

This paper focuses on roadside freight surveys. Its objective is to examine whether general purpose roadside freight surveys can be improved by the widespread or even systematic inclusion of new questions in the survey forms. The paper also examines whether the additional information is worth the additional cost associated with it.

Before proceeding to present the methodology, let us briefly discuss the scientific value of such efforts. From a microeconomic perspective, road freight carriers are producers which transform inputs (vehicles, energy, working force) into outputs (transport operations). The relationship between the inputs and the outputs is referred to as the technology, and its structure is most often assumed to be very simple: the amount of inputs necessary to carry a given amount of tons of freight for a certain distance is assumed to be proportional both to the weight of the freight and to 
the distance to be covered. In other words, the inputs are perfect complements, and it is thus legitimate to describe road transport by a per ton, per kilometer unit cost (with some refinements, e.g. distinction between commodity groups). While this assumption may prove sufficient for many purposes, a more realistic representation of the structure of freight road transport costs is desirable in some cases. This is especially true when addressing issues such as the regulation of truck weight and size, the conversion of commodity flows into traffic in freight transport demand models, and mode choice. Three of the potential difficulties that arise when trying to examine in detail the structure of costs of road freight transport are listed below.

First, road freight transport services are produced using an infrastructure network and fixed inputs (vehicles). This has a direct impact on the productivity of freight carriers, and thus on freight rates. For example, on a given origin-destination pair, main haul and back haul freight rates are closely related [2]; these spatial dependencies become increasingly complex when more sophisticated network structures are considered [3]. This is closely related to the issue of empty return [4].

Second, road freight transport operations take place in a logistical context, which is an issue because the microeconomic drivers of logistical decisions are at present only partially understood [5]. In particular, shippers' choices about shipment sizes and transport modes are highly dependent on freight rates, and particularly on the relationship between shipment size and freight rates, which is not linear. Conversely, freight rates are influenced by the choices of shipment sizes made by shippers. For a discussion on the microeconomics and econometrics of the shipment size choice, and more generally on the introduction of logistical principles in freight transport modelling, see e.g. [6, 7]

Third, carriers optimise their productivity by grouping shipments whenever possible. Assigning shipments to vehicles so as to maximise the average loading factor of those vehicles is known to be a complex problem of operations research (the bin-packing problem), and it has a complex impact on the prices of freight transport $[8,9]$. In addition, vehicle capacity is an important instrument of freight transport policy and, as such, has been the object of econometric investigations (see e.g. $[10,11])$. Note that vehicle capacity is often measured in tons, whereas the relevant unit varies a lot with the context: it can indeed be tons, but also $\mathrm{m}^{3}$, pallets, etc.

The study of these issues requires accurate observation and knowledge of road freight transport. In particular, road freight transport surveys need to be improved whenever possible to provide relevant information, especially as regards the constraints under which road freight carriers operate, and the way these constraints influence their productivity. The additional information obtained is useful from a theoretical perspective, as it allows a better qualitative understanding of road freight transport; from a modelling perspective, since improved data offer an opportunity to improve models; and from a decision support perspective, insofar as the capacity of a stakeholder to assess the effects of a decision is determined by the accuracy and quality of the data at hand.

Within the wide range of road freight surveys, the characteristics of roadside freight surveys are particularly interesting. First, they are widely used, and yield a lot of data. Second, given the structure of the costs involved in implementing a roadside survey, the incremental cost of adding a few extra questions to existing forms is fairly small, provided they can be asked and answered quickly. Third, they involve face-to-face contacts between interviewers and truck drivers. As such, they generally obtain excellent response rates, and, more importantly, they offer special opportunities to obtain information on road freight transport operations: truck drivers are of course not freight transport managers, but they know more about the transport operations they are involved in than what they are usually asked about.

In investigating potential opportunities to improve roadside freight surveys, this paper employs the following methodology: a number of new questions were designed and assessed through two dedicated surveys: a pretest survey and a confirmation survey. Roadside surveys are generally used to gather combined information on vehicles (number of axles, vehicle type), trips (origin, destination, length), and commodities carried (commodity type, weight, etc.). The new questions tested in this study addressed four topics that do not usually fall within the scope of roadside freight surveys: first, the volume capacity constraint of vehicles; second, the way road freight carriers organise their operations; third, the logistical context in which these operations take place, more precisely whether or not there is a specific arrival time imperative; fourth, the locations and durations of the breaks taken by drivers. On the basis of the outcome of the pretest survey, in they were first tested, the information obtained from the questions on volume constraint and the organisation of freight transport operations were deemed most fruitful. These conclusions were tested again during the confirmation survey. 
The outline of this study is as follows: current international practice for roadside freight surveys is discussed in Section 2, with a description of the usual contexts, objectives and methodologies. Section 3 presents the design of the pretest survey, and its results. The outcome of the confirmation survey is presented in Section 4. Finally, conclusions are drawn in Section 5: the concrete consequences in the field of road freight transport modelling and decision support, and the potential for extension to other types of surveys are discussed.

\section{Roadside freight surveys: methodology and usage}

Roadside freight surveys are one of the main types of survey used for the observation of road freight transport. They are very widely used, but often stem from local initiatives, and they are seldom managed at national level (unlike, for example, the North-American Commodity Flow Survey, which is a shipper-based survey; or the European national road freight surveys, which are carrier based, with methodologies and data harmonised by Eurostat $[12,13])$. However, it is possible to obtain a decent picture of current practice and usage in roadside freight surveys from a number of thorough reviews undertaken during the past few years. The following three references constitute the basis for the discussion: the North-American synthesis 410 on Freight Transportation Surveys, undertaken as part of the National Cooperative Highway Research Program (NCHRP), under the administration of the Transportation Research Board [1]; the report produced under the Green Logistics project, financed by the UK governement agency EPSRC (Engineering and Physical Science Research Council), which reviews freight surveys in urban contexts in Europe [14, 15]; and an international review of freight data collection methods for Transport Canada [16]; this last report reviews fewer surveys than the other two, but covers more countries.

A roadside survey consists of one or several data collection points, located at strategically chosen locations, matching the specific objectives of the survey. At each of these data collection points, trucks are drawn randomly from the traffic flow, and pulled over to an area where drivers can be interviewed. Subsequently, two conditions at least are necessary for a roadside survey: first, the cooperation of the police is required to pull over vehicles from the traffic; second, facilities where interviews can be held are needed (e.g. highway parking, or service areas). Once the vehicles are stopped, the interviewers can gather information: first, by direct observation of the vehicle; second, by interviewing the driver. To minimise driver downtime, the interview should be short (no more than a dozen questions). There can be several interviewers at a data collection point, in order to increase throughput. The advantage of roadside freight surveys are, as stated above, firstly that they generally provide an excellent response rate and secondly that in many countries they are one of the only ways to obtain data on international transit traffic.

A few remarks need to be made: first, in most cases, roadside surveys take place during the day, and therefore miss out night traffic. Second, the survey protocol described in this paragraph is sometimes referred to as the "roadside interview survey" protocol, considered as a subcategory of the more general "roadside survey" category, which also encompasses plate, roadside handout, and roadside handout interview surveys [17]. Third, there exists another type of survey, called the parkside survey. In a parkside survey, truck drivers are interviewed in a service area during their breaks. It is very similar to the roadside survey protocol, except that it does not require the cooperation of the police, and is not subject to the same time constraints as roadside surveys, so that longer survey forms can be administered. However, the drivers are not drawn randomly from the traffic so this survey methodology introduces sampling biases. Finally it should be noted that in terms of cost per observation, roadside freight surveys are relatively expensive.

The immediate purpose of roadside freight surveys is most often to examine commodity flows and vehicle trips together, with the purpose of building origindestination matrices. These surveys most often stem from local or regional data needs, to study commodity and vehicle flows within an urban area, through a tunnel, a border, or a road or set of roads. There have been some national roadside freight surveys, such as the NRS (National Roadside Surveys) coordinated by the Canadian Council of Motor Transport Administrators in 1991, 1995 and 1999 [16]. Roadside surveys can also serve specific objectives, such as to collecct specific information for modelling purposes. For example, in [18], roadside freight surveys were carried out to calibrate vehicle to commodity ratios, with a focus on vehicle types and average loads. In [19], roadside freight survey data are used to estimate departure times. There is no systematic management and grouping of roadside freight surveys at national level. In France for example, the Geode information system groups most roadside 
surveys (including passenger roadside surveys), but the data are not completely harmonised.

The data gathered during roadside surveys can be categorised into two groups: trip-related data and freight-related data. Although survey forms do vary, they correspond by and large to the following grid:

- Number of axles. Observed by the interviewer, generally between 2 and 5 .

- Vehicle type. Also observed by the interviewer, who identifies a type within a typology. The typology varies with the survey.

- Trip origin. The driver is asked his last compulsory stop, whether is was to load or unload freight or to pick up the vehicle. Note that the two possibilities are distinguished when asking the question, because the origin of the freight's movement can be different from the origin of the driver's trip.

- Trip destination. The driver is asked his next compulsory stop, whether it is to load or unload freight, or to collect the vehicle.

- Trip length. The interviewer asks the length of the trip. The driver's answer is sometimes approximate.

- Empty or loaded. The interviewer asks whether the vehicle contains freight or not.

- Commodity type. In the case of a loaded vehicle, the interviewer asks the nature of the freight. It should be noted that when the semi-trailer holds a container, the commodity type is generally unknown to the driver. In other cases, the driver generally bears a document (the waybill) which describes the freight, the pickup and delivery times, as well as the route. If there is more than one commodity, different approaches can be chosen. A preferred approach is to ask the driver the type of the main commodity.

- Freight quantity. The driver is asked how many tons of freight he or she is carrying. This data is also available on the documents accompanying the freight.

- Hazardous materials. There can be questions regarding specifically hazardous materials.

This list of questions may vary with the circumstances. In some cases, the interviewer asks the driver the type of premises at the origin and the destination of the trip (offices, plant, warehouse, port, etc.). In addition, in urban conditions, the unit defining the quantity of freight may be left to the choice of the driver (weight, volume, pallets, boxes, etc.)

Clearly, this type of survey form yields useful and relatively accurate information (provided that the drivers' declarations are correct) on both trips and commodity flows. However, a roadside survey offers opportunities for direct contact with truck drivers, who may know more about the transport operation they are participating in than what they are asked. This is considered below.

\section{Design of the new survey form and pretest survey}

In order to identify ways in which roadside surveys can be improved to yield more information about road freight transport, a specific survey form, addressing new topics, was tested as part of a specific survey. These topics are: the volume constraint of vehicles, the organisation of road freight transport operations, the existence of a specific arrival time imperative, and the durations and locations of the breaks taken by drivers. The methodology and outcome of this approach are presented here.

A general description of the survey is given in Section 3.1. The new questions are then described and discussed: volume constraint in Section 3.2, transport organisation in Section 3.3, arrival time imperatives in Section 3.4 and location and duration of breaks in Section 3.5. The survey form itself is shown in Fig. 1. It is an extended version of the standard French roadside survey form. The standard questions are Q1-Q6, and Q8-Q10. They are consistent with the list of questions given in Section 2. The other questions were specific to this study.

\subsection{The RN10 survey}

The survey took place between 4th December and 12th December 2007 some $50 \mathrm{~km}$ from Bordeaux, France. The survey is named after the road on which it was located: the RN10, one of the main routes between Bordeaux and Paris. This means that the traffic surveyed was mainly interurban. Both traffic directions were surveyed. Because the police were not involved, the roadside protocol could not be strictly applied. As a consequence, the drivers were interviewed on a nearby service area during their breaks. This has no impact on the analysis that follows, which focuses on the quality and relevance of the information obtained through the new questions. However, the figures presented below are most probably biased, as a consequence of the survey methodology. 693 truck drivers were interviewed, 686 of these interviews yielded workable data.

Before discussing the information obtained from the additional questions, the sample is briefly described. $89.8 \%$ of the vehicles in the sample have 5 axles. 


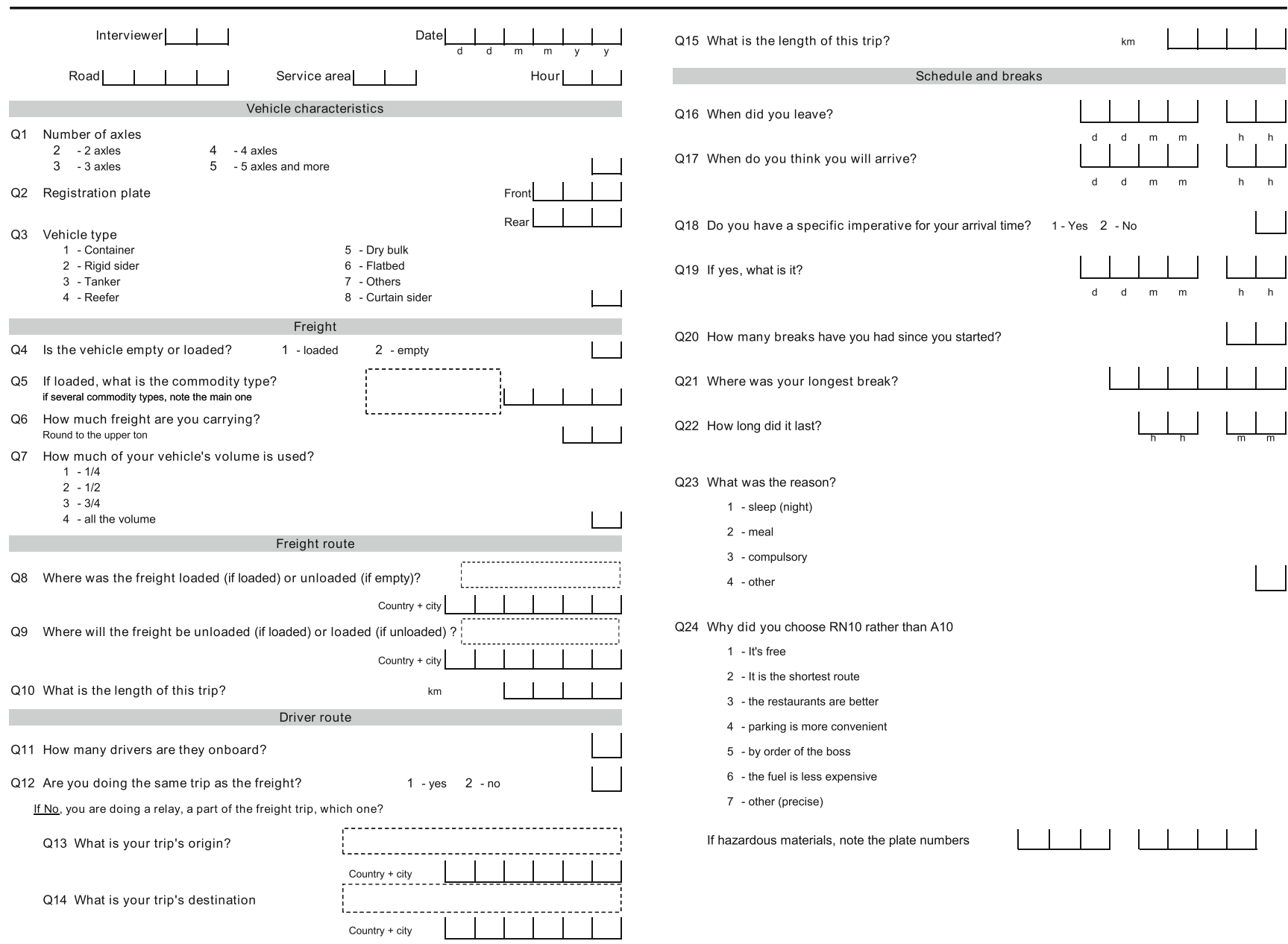

Fig. 1 RN10 survey form (2 pages, translated from French by the authors)

The survey being located on a main road between two big cities, and on a major international freight traffic route, notably between France and the Iberian peninsula, the majority of vehicles could be expected to be large. For the same reason, various nationalities are observed among the vehicles: $45.5 \%$ are French, $24.6 \%$ are Spanish and $20.4 \%$ are Portuguese. With respect to their origins and destinations, $42 \%$ of the trips are national, $58 \%$ international. $56.9 \%$ of the international trips are transit trips.

The vehicle types closely mirror the number of axles. The trucks with 2 axles are mainly reefers or tautliners, the 3 and 4 axles are mainly flatbeds or dry bulk. About half of the 5 axle vehicles are tautliners. The other types are by and large equally distributed, except for the containers, which are rare. Tautliner vehicles can be considered as general purpose vehicles, while the other vehicles are used for commodities with specific constraints (handling, temperature, safety, etc.). The use of specific equipments in road freight transport is thus significant. They meet specific requirements, as indirectly illustrated by the average length of trips per vehicle type (Table 1). The distribution of vehicle types is relatively similar in both directions.

The commodity type is encoded under NST/R (the 1970 revised European standard goods classification for transport statistics, replaced by a the new NST classification in 2007 [20]). It is observed for $86 \%$ of the loaded vehicles. Interestingly enough, while

Table 1 Length of trips per type of vehicle

\begin{tabular}{lcc}
\hline Vehicle type & $\begin{array}{l}\text { Freq. } \\
(\%)\end{array}$ & $\begin{array}{l}\text { Average } \\
\text { distance }(\mathrm{km})\end{array}$ \\
\hline Container & 1.02 & 883 \\
Rigid sider & 12.39 & 1,042 \\
Tanker & 7.73 & 658 \\
Reefer & 11.52 & 1,554 \\
Dry bulk & 10.06 & 310 \\
Flatbed & 9.62 & 973 \\
Curtain sider & 46.49 & 1,428 \\
Others & 1.17 & 1,655 \\
Total & 100.00 & 1,176 \\
\hline
\end{tabular}


commodity flows appear to be symmetrical when commodity types are examined at the most aggregate level (10 categories), this symmetry is broken when commodity types are considered at the most detailed level (176 categories in the NST of 1970). A breakdown of trips per commodity type is given in Table 2, together with the average values for the lengths of the corresponding trips.

As noted in this table, $10.8 \%$ of the vehicles surveyed were running empty. However, this percentage closely reflects the type of vehicle. Some vehicles, such as tautliners, are quite versatile, and present empty running factors of less than $10 \%$. More specialised trailers, such as tankers and dry bulks, have much higher empty running factors, in excess of $20 \%$. Finding backhaul freight is more difficult for these specialised vehicles.

It would be possible to conduct any number of analyses of this kind. However, that is not the purpose of this study. The points specifically addressed by the survey form will now be examined. To ensure minimum homogeneity in the transport operations analysed, the discussion that follows is confined to the 5 axle vehicles, which represent about $90 \%$ of the sample.

\subsection{Volume constraint}

Weight capacity is a major technological constraint in road freight transport. The ability of carriers to fill their vehicles to capacity is a critical driver of their productivity. It is measured by the loading factor, i.e. the ratio of the weight of freight carried to vehicle capacity. This ratio is used in almost all spatialised models to convert commodity flows into vehicle flows, (for example, the loading factor was chosen as one of the key factors of freight transport demand in the European REDEFINE project [21]), and more generally to assess the average

Table 2 Length of trips per commodity type

\begin{tabular}{lcc}
\hline Commodity type & $\begin{array}{l}\text { Freq. } \\
(\%)\end{array}$ & $\begin{array}{l}\text { Average } \\
\text { dist. }(\mathrm{km})\end{array}$ \\
\hline 0 - Agri. products & 10.20 & 1,451 \\
1 - Foodstuff & 14.14 & 1,235 \\
2 - Solid mineral fuels & 0.00 & - \\
3 - Petroleum products & 3.94 & 908 \\
4 - Ores and metal waste & 2.48 & 1,230 \\
5 - Metal products & 2.19 & 1,203 \\
6 - Minerals, building mat. & 8.89 & 406 \\
7 - Fertilizers & 0.58 & 698 \\
8 - Chemicals & 6.41 & 1,228 \\
9 - Machinery, etc. & 37.17 & 1,482 \\
NA & 3.21 & 650 \\
Empty & 10.79 & 427 \\
Total & 100.00 & 1,176 \\
\hline
\end{tabular}

productivity of road freight transport and of its evolution over time.

Freight weight can be obtained fairly accurately by roadside surveys, since it is shown on the documents which accompany the freight. Vehicle capacity is also easily observable, and if not observed, can be deduced more or less precisely from the number of axles. Therefore, the loading factors of the vehicles surveyed are easily calculated; the influence of the weight constraint on the organisation and costs of road freight carriers can be assessed.

However, another constraint limits the productivity of carriers: the volume constraint. Unlike the loading factor, this constraint is usually ignored in transport statistics. As a result, its influence on road freight transport costs is not well understood.

The reason that volume constraint is difficult to measure is that, for a given vehicle, neither the volume of the freight nor the volume of the vehicle are easy to observe. In particular, neither can be obtained from an interview with a truck driver. However, even if truck drivers do not know the exact volume of their freight in $\mathrm{m}^{3}$, they are aware of the approximate volume occupied by the freight in their vehicle, and can tell if the vehicle is, say, half empty, or two thirds full. Drivers were therefore asked a specific question in the RN10 survey: if the vehicle was not empty, the interviewer asked the driver if a quarter, a half, three quarters or the totality of the vehicle's volume was used (question Q7). It would seem that the drivers understood the question correctly, and were always able to answer it in the RN10 survey.

The question is whether this additional information is useful: in other words, do the volume and weight constraints play the same role? If that were the case, then measuring both variables would be a waste of time and resources. Consider Table 3: two constraints are considered; a weight constraint and a volume constraint. If there are 24 tons or more of freight in a vehicle, then the vehicle is considered full in weight in Table 3. If, according to the driver, the vehicle is full in volume, then it is indicated as such in Table 3. From the examination of these two variables, both constraints appear to play important roles; what is more, these roles are clearly distinct. In particular, increasing the

Table 3 Weight and volume constraints, RN10 survey

\begin{tabular}{llll}
\hline & & Full volume \\
\cline { 3 - 4 } & & No $(\%)$ & Yes $(\%)$ \\
\hline Full weight & No & 23.2 & 42.0 \\
& Yes & 8.6 & 26.1 \\
\hline
\end{tabular}


weight limit of vehicles while leaving the volume limit unchanged would impact only $8.6 \%$ of the vehicles in the sample, compared with $42.0 \%$ for a change in the volume limit with a constant weight limit.

The relationship between weight and volume is shown in Fig. 2, where the area of each circle is proportional to the number of vehicles concerned, the x-axis being the load factor (between 0 and 1) and the y-axis the proportion of the vehicle's volume occupied by the freight (also between 0 and 1 ). This, combined with the correlation of 0.16 between the freight weight and the freight volume for loaded vehicles, implies that the load factor in weight alone is not a complete measure of the constraints and productivity of road freight transport.

While the figures given in this section should be treated carefully, due to the possible bias resulting from the survey protocol, approximately measuring the volume used in the vehicles appears to be both feasible in general purpose roadside freight surveys, and useful with regard to the importance of the variable. Such data can be a useful basis for studies of truck size and weight, in which one difficulty is to evaluate the elasticities of road freight transport demand with respect to weight limits and vehicle dimensions. The results illustrated in this section, although approximate, also explain why increasing usable volume while keeping the outer dimensions unchanged is as much a major axis of innovation of the trucking industry as reducing the curb weight of vehicles. They advocate for a more comprehensive representation of the productivity of road freight transport, which is at best imperfectly accounted for by considering an average load factor in tons alone. Finally, the role of commodity type should not be let aside; for certain commodity types, for example liquids, increasing the volume of a vehicle is absolutely useless if the weight constraint remains unchanged.

Note that the measurement of volumetric occupancy is compulsory in the statistics on the carriage of goods by road for European Union member states and candidate members [12]. The databases specifically affected

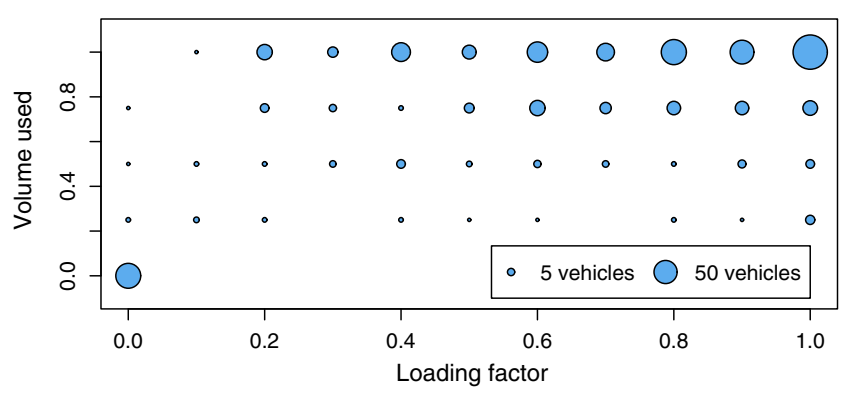

Fig. 2 Loading factor and volume used, RN10 survey by this rule are the national freight surveys addressed directly to carriers, which are sampled using national vehicle registries (and therefore do not necessarily include observations of all trips). The carriers have to state for each trip whether the vehicle was empty, less than $90 \%$ full, or more than $90 \%$ full, in terms of volume. However, it would not seem the data thus obtained is always used to measure the productivity of road freight transport. The discussion above concerning the importance of this issue applies to these databases too.

\subsection{Transport organisation}

From a microeconomic perspective, road freight transport is not seen as a complicated technology. The two inputs, vehicles and drivers, are generally assumed to be perfect complements: they must be used in fixed proportions to produce a given amount of transport, measured in vkm (vehicle-kilometre). The cost function of road freight transport is proportional to the $\mathrm{vkm}$ output, and the speed of road freight transport is fully determined by the speed limitations and the rules determining the breaks drivers are obliged to take. The complementarity of drivers and vehicles as inputs in the production function is confirmed econometrically. For example, by fitting statistically a constant elasticity of substitution production function with the number of vehicles and drivers as its arguments, [22] found that the elasticity of substitution between these two factors is 0.2 , which is low.

The reality is more complex. Road freight carriers have the option to organise transport operations in order to decrease travel times for long trips. Within the framework of a long trip (longer than about ten hours), the driver has to stop for at least eight hours in order to sleep. During that break, the vehicle is obviously stationary. Carriers have two ways of overcoming this constraint. The carrier can decide to put two drivers in a truck, so that one of them drives while the other one sleeps. A more complex approach is also possible: if there is a very regular flow from a given starting point to a given destination, the carrier can synchronise the movements of several drivers and vehicles so that each vehicle is always on the move and each driver is at the wheel for the regulation time. The reduction in travel time comes at a price of increased labour requirements.

These two methods of organisation are easily identified during a roadside survey: the feature of a double crew is simply that there are two drivers or more on board; while a relay is characterised by the fact that the driver and the freight do not have the same starting point and destination. They are thus identified 
by questions Q11 to Q15 in the RN10 survey form. Note that the authors could identify one survey in which the number of drivers was observed [23]. However the data was not analysed and relays were not observed.

Except in two cases, all trips with a double crew or a relay system were observed with 5 axle vehicles. Their frequencies are given in Table 4, as well as the corresponding average distances and speeds. These frequencies are low: it seems that double crew and relay systems account for a limited share of the traffic. These figures should be treated with care, given the sampling conditions of the survey; however, they are of similar orders of magnitude as those obtained in the confirmation survey described in Section 4.

Departure and arrival times were observed (questions Q16 and Q17 of the survey form), so travel durations and speed were calculated. The average trip length and speed corresponding to each type of organisation are indicated in Table 4. The double crew and relay organisations allow for higher average speeds. They are, of course, encountered on long trips. From an economic standpoint, double crew and relay arrangements are expected to be found on long distances and for time-critical or high depreciation cost goods, such as agricultural products and foodstuff. This is not disproved by the comparison (not specified here) of commodity types with transport arrangements.

\subsection{Imperative arrival times}

The increasing role of logistical imperatives in the recent development of freight transport markets has been discussed at length. However, the notion of logistical imperatives itself is not precisely defined, and can take many forms. These include the ever increasing importance of the customer preferences in shippers' logistical arrangements, the closer integration between logistical decisions and production and marketing decisions, and the rationalisation of logistical and transport operations.

The issue addressed in this section is whether it is possible to assess the influence of these phenomena on road freight transport by means of roadside surveys. Two questions were introduced into the RN10 survey form to explore this possibility. The interviewers asked

Table 4 Organisation of transport operations, RN10 survey

\begin{tabular}{llll}
\hline Organisation & Freq. $(\%)$ & Average dist. $(\mathrm{km})$ & Speed $(\mathrm{km} / \mathrm{h})$ \\
\hline Simple crew & 93.1 & 1,182 & 33.9 \\
Double crew & 5.4 & 2,143 & 40.6 \\
Relay & 1.5 & 1,606 & 50.8 \\
\hline
\end{tabular}

the drivers to give both their expected arrival times and imperative arrival deadlines, making a clear distinction between these two notions (questions Q18 and Q19 of the survey form).

The answers are quite instructive, but not fully as expected. Indeed, for the 5 axle vehicles, most drivers $(77.0 \%)$ responded that they had no arrival deadline. $6.9 \%$ of them answered they had an arrival deadline, which was in fact their expected arrival time. Incidentally, many drivers resented the question, on the grounds that it undermined their autonomy. Ultimately, $16.2 \%$ of the drivers provided an arrival deadline that was clearly distinct from their expected arrival time. For these drivers, the margin between the expected arrival time and the deadline correlated positively with the length of the trip.

Overall, the existence and definition of logistical imperatives for road freight carriers are not striking factors. Road freight carriers organise their operations with sufficient time margins to offer shippers the expected level of service associated with the transport operation.

The contribution that roadside freight surveys can make to the issue of the logistical imperatives under which carriers operate is not straightforward. The trade-off between direct transport costs and other service quality parameters, such as speed or travel time reliability, is a very interesting issue, but may be beyond the scope of what can be reasonably expected from general purpose roadside freight surveys. This matter seems to require more specific data collection protocols (an example of an application with Intelligent Transport Systems is given by [24]), or modelling and estimation (in [19] the authors estimate the difference between desired and actual departure times for carriers).

\subsection{Location and duration of breaks}

So that drivers remain alert and drive safely, breaks are enforced by law. This law describes precisely when breaks must be taken, and how long they should last. In consequence, drivers have little choice but to comply with the regulations.

At least two important issues of transport policy depend closely on the regulation of breaks. The first one is the effective speed of trucks, which directly determines the productivity of road freight transport (as well as the costs of wear and tear on road infrastructure, and the probability of accidents). Moreover, as already discussed in Section 3.3, motor carriers can introduce specific arrangements to increase the effective speed of trucks while complying with the law. 
The second issue is the congestion of service areas on highways and major roads. Breaks being compulsory, if a driver does not find room on a service area, he or she will park elsewhere, including by the side of the road, with all the predictable consequences in terms of safety and risk of robbery. These issues may induce drivers to reconsider their route choice. For this reason, it is both an important matter for infrastructure operators and from the perspective of a road infrastructure planning.

The usefulness of roadside surveys in investigating these effects was tested in the pretest survey. Drivers were asked how many breaks they had taken since their departures, the locations and durations of their longest breaks, and the reason for those breaks (questions Q20 to Q23). Unfortunately, their answers yield little useful information, and are not presented here in detail. Indeed, apart from choosing the place where they can park, drivers have little freedom of choice regarding their breaks. The data only illustrates the rules they follow, which themselves stem directly from the law.

A dedicated geographical approach, in which the route and parking choices of drivers were analysed together with the occupancy rates of rest areas would probably be an interesting direction of research. However, the cost-benefit ratio of including these questions in all roadside freight surveys would seem too large. Specific surveys are certainly more relevant with regard to this particular topic.

\section{Final survey form and confirmation survey}

As shown in the previous section, the questions concerning the volume constraint and the organisation of transport operations are those which yielded the most useful results, and which might potentially be usefully extended to all roadside freight surveys. To confirm these results within the framework of a standard roadside survey protocol, a confirmation survey was carried out with a reduced version of the RN10 survey form, comprising only questions Q1-Q12.

The RN10 survey took advantage of unusual survey conditions to test a fairly large number of new questions. In particular, the fact that the RN10 survey consisted of interviews of drivers during their breaks on a rest area meant that a long survey form could be administered. In standard roadside freight surveys, drivers are interrupted during their trips, so the whole interview should not last more than five minutes, limiting the survey form to around a dozen questions.

The confirmation survey took place between 14th May and 20th May 2008, on a number of roads between the cities of Limoges and Poitiers in France,
Table 5 Weight and volume constraints, A10-A20 survey

\begin{tabular}{llll}
\hline & & Full volume & \\
\cline { 3 - 4 } & & No $(\%)$ & Yes $(\%)$ \\
\hline Full weight & No & 28.0 & 34.4 \\
& Yes & 11.8 & 25.7 \\
\hline
\end{tabular}

including the A20 highway in the north-south direction near Limoges and the parallel A10 highway between $100 \mathrm{~km}$ and $200 \mathrm{~km}$ to the west. In addition to the standard questions in the French roadside survey form, the following questions were included:

- How much of the vehicle's volume is used?

- How many drivers are there on board?

- Is the driver making the same trip as the freight?

The sample obtained by this survey consists of 630 vehicles. $75 \%$ of them were 5 axle vehicles, the average trip length was $514 \mathrm{~km}$, and $21.9 \%$ of the vehicles were running empty. This sample is not directly comparable to the previous one: the traffic surveyed is more local and disparate than that observed in the RN10 survey.

Despite these dissimilarities, the conclusions drawn in Section 3.2 are confirmed. Table 5, which corresponds to the loaded 5 axle vehicles, shows the respective roles of the weight constraint and of the volume constraint in the sample. It is consistent with Table 3.

Similarly, double crew and relay systems are identified. The results are given in Table 6. Again, double crew and relays appear relatively rare, and trips are longer on average with double crew or relay systems. However, the rankings are reversed: double crews are much less frequent in this sample than in the RN10 sample; this might be due both to the distinct survey protocols and to the fact that the types of traffic differ in nature. Note that the average speeds obtained from the RN10 survey could not be confirmed, as no information was available on departure and arrival times.

On the whole, the confirmation survey validates the potential of the questions on the volume constraint and the organisation of transport operations as valuable extensions to the standard roadside freight survey forms, yielding new information for a limited additional cost.

Table 6 Organisation of transport operations, A10-A20 survey

\begin{tabular}{lll}
\hline Organisation & $\begin{array}{l}\text { Frequency } \\
(\%)\end{array}$ & $\begin{array}{l}\text { Average } \\
\text { distance }(\mathrm{km})\end{array}$ \\
\hline Simple crew & 92.7 & 572 \\
Double crew & 1.9 & 829 \\
Relay & 5.3 & 735 \\
\hline
\end{tabular}




\section{Conclusion}

The roadside survey is a widely used data collection protocol to obtain information on road freight transport. During a roadside survey, vehicles are diverted from the traffic and their drivers are interviewed. The variables observed are usually vehicle type, the starting point, destination and length of the trip, and the nature and quantity of the commodities transported. Often addressing local or regional requirements, the main purpose of roadside surveys is generally to obtain origin-destination data.

Roadside surveys are distinct from other types of road freight surveys insofar as they offer the opportunity for direct contact with truck drivers. Although truck drivers are not road freight managers, it is nevertheless possible that standard roadside surveys do not make the most of what they know about the transport operations in which they participate. The objective of this paper is to find out whether certain new questions could be introduced widely, even systematically, in roadside freight surveys, in order to obtain a better understanding of road freight transport.

In order to determine how roadside surveys can be enhanced, an original, comprehensive survey form was developed and tested. Apart from the standard questions, four additional factors were explored: freight volume, transport organisation, the presence of specific logistical imperatives, and driver breaks. For each of these topics, drivers were asked a number of specific questions, to determine whether or not these questions would be useful additions to roadside freight survey forms. These results were confirmed with another survey.

As regards the volume constraint, from the results of the two surveys we can conclude that useful, albeit approximate, information can be obtained from drivers. This information is important since the volume constraint seems to play a role that is at least as important as the weight constraint in determining the productivity of carriers. Given that freight transport increasingly concerns high added value commodities, with a lower density than heavy industrial goods, one can expect this constraint to play an ever increasing role in the future. This issue should be considered closely: both modelling issues, such as the conversion of commodity flows into vehicle flows, and transport policy issues, such as in particular the influence of truck size and weight regulations, require such data for a quantitative assessment; and until accurate information on the volumes of shipments and vehicles is made systematically available, roadside freight surveys can be a useful source of data.
The organisation of transport operations is also a topic on which roadside freight surveys can yield useful information at a small additional cost. By observing how many drivers are on board, and by asking drivers whether they are making the same trip as the freight, it is possible to identify double crew and relay systems. Comprehensive knowledge of these practices is currently lacking, and yet they play a critical role in the productivity of road freight carriers, and in determining the ranges of services they are able to provide to their customers. Such data would usefully contribute to our knowledge of freight transport, both from a qualitative standpoint and from the perspectives of modelling and public policy decision support.

Two other potential areas of improvement were investigated. The first one concerned the existence of logistical imperatives, specifically deadlines for arrival. The second concerned the locations and durations of breaks. Neither performed very well. Including these questions specifically in general purpose roadside freight surveys would not be helpful. The subjects themselves are of interest, but they would probably require dedicated survey protocols.

The surveys presented in this study have a major shortcoming. No distinction has been made between public and private carriers. It is certainly possible to ask drivers this question, which is highly relevant, since public and private road freight transport are clearly distinct sectors. This is also a recommended improvement of roadside freight surveys.

The objective of this paper was to investigate the possibility of extending roadside survey forms so that they provide useful additional information on freight transport. This possibility would appear to be significant. Moreover, to a certain extent, there is a possibility that the conclusions presented in this paper might also apply to other types of surveys, especially national road freight surveys aimed at carriers. While these surveys do not offer the same opportunity as roadside surveys to obtain simultaneous information on origin-destinations and routes, and exclude by design the international transit trips, they are still a useful and widely used source of information on road freight transport. Extending these surveys along the lines presented in this paper could be a potential opportunity to improve our knowledge of freight transport.

Acknowledgements We would like to sincerely thank Pierre Baillet and Yves Saige from Cété Sud-Ouest, for their support and help, and for offering us the opportunity to experiment with the two roadside surveys this study is based upon. We would also like to thank Elisabeth Windisch and Vincent Benezech for their advice and help. We would also like to express our sincere 
gratitude to two anonymous referees, whose commentaries helped us to improve the paper substantially.

Open Access This article is distributed under the terms of the Creative Commons Attribution License which permits any use, distribution and reproduction in any medium, provided the original author(s) and source are credited.

\section{References}

1. Transportation Research Board, NCHRP Synthesis 410, Freight Transportation Surveys (2011) A synthesis of highway practise

2. Felton JR (1981) Impact of ICC rate regulation upon truck back hauls. J Transp Econ Policy 15(3):253-267

3. Jara-Díaz S (2007) Transport economic theory. Elsevier

4. Holguín-Veras J, Thorson H (2003) Modeling commercial vehicle empty trips with a first order trip chain model. Transp Res Part B 37:129-148

5. Combes F (2010) Logistic imperatives and mode choice. In: Proceedings of the European transport conference, Glasgow, Scotland, UK

6. Combes F (2009) The choice of shipment size in freight transport. Ph.D. thesis, Université Paris-Est

7. Tavasszy L, Ruijgrok K, Davydenko I (2010) Incorporating logistics in freight transportation models: state of the art and research opportunities. In: Proceedings of the world conference on transport research, Lisboa, Portugal

8. Verheijen B (2010) Vendor-buyer coordination in supply chains. Ph.D. thesis, Erasmus University Rotterdam

9. Combes F (2010) On shipment size and freight tariffs: technical constraints and equilibrium price schedules. In: Proceedings of the 89th meeting of the Transportation Research Board, Washington, USA

10. McKinnon AC (2005) The economic and environmental benefits of increasing maximum truck weight: the British experience. Transp Res Part D 10:77-95
11. Bereni M, Jacob B (2009) A look into the likely consequences of adapting weights and dimensions of heavy commercial vehicles in Europe. Transportation Research Board, Washington

12. Eusrostat (2008) Road freight transport methodology. Reference manual for the implementation of Council Regulation No 1172/98 on statistics on the carriage of goods by road

13. Eurostat (2008) Methodologies used in surveys of road freight transport in member states and candidate countries

14. Allen J, Browne M (2008) Review of survey techniques used in urban freight studies. University of Westminster, London, UK

15. Allen J, Browne M (2008) Survey forms used in urban freight studies. University of Westminster, London, UK

16. Haider M, Ewing G, Patterson Z (2008) Freight data collection methods, a review of existing methods

17. Center for Urban Transportation Research (2006) Development for revised methodology for collecting origindestination data. University of South Florida

18. Nuzzolo A, Crisalli U, Comi A (2009) A demand model for international freight transport by road. Eur Transp Res Rev $1: 23-33$

19. Russo F, Vitetta A, Comi A (2009) Estimation of target time distribution for agri-food products by road transport. In: Wilson NHM, Nuzzolo A (eds) Schedule-based modeling of transportation networks, theory and applications. Springer

20. Commission Regulation (EC) (2007) No. 1304/2007. Off J Eur Union L290:14-16

21. NEI et al (1999) REDEFINE: relationship between demand for freight-transport and industrial effects. Summary report

22. Delvaux G, Duhautois R (1998) Complémentarité des facteurs de production dans le transport routier de marchandises en France. Notes de Synthèse du SESP

23. City of Edmonton, Alberta Transportation (2004) Edmonton region external truck/commodity survey, revised edition. Originally published in 2003

24. Ando N, Taniguchi E (2005) An experimental analysis on probabilistic vehicle routing and scheduling with ITS. J East Asia Soc Transp Stud 6:3052-3061 